\title{
Micelle-Sensitized Constant-Energy Synchronous Fluorescence Spectrometry for the Simultaneous Determination of Pyrene, Benzo $[a]$ pyrene and Perylene
}

\author{
Li-Fang HE,*** Dan-Li LIN,** and Yao-Qun LI*** \\ * Department of Chemistry, Longyan College, Longyan 364000, China \\ **Department of Chemistry, The MOE Key Laboratory of Analytical Sciences, Xiamen University, \\ Xiamen 361005, China
}

\begin{abstract}
The use of micellar media in constant-energy synchronous fluorescence spectrometry has been proposed. The influence of some aqueous micellar systems on the determination of pyrene, perylene and benzo[ $a]$ pyrene has been investigated. The presence of these micellar systems allows their determination in aqueous media, thus avoiding the use of an organic solvent, and greatly enhances the fluorescence signals. The combination of a constant-energy synchronous scanning technique and a micellar system provided a single spectrum for the simultaneous identification and quantitative determination of the three polycyclic aromatic hydrocarbons (PAHs). Further there was no energy transfer among them, making the measurement simple and fast. A constant-energy difference of $2800 \mathrm{~cm}^{-1}$ was selected. The analytical characteristics of the proposed method in the presence of anionic micelles of sodium dodecylsulfate (SDS) were studied. The detection limits were at a level of $\mathrm{ng} \mathrm{ml}^{-1}$. Analysis of water samples from two different origins spiked with known amount of pyrene, perylene and benzo[a]pyrene also gave satisfactory results, and total average recoveries were greater than $97.1 \%$.
\end{abstract}

(Received October 12, 2004; Accepted January 21, 2005)

\section{Introduction}

The identification and quantification of polycyclic aromatic hydrocarbons (PAHs) in the environment are of great interest due to their carcinogenic and mutagenic characteristics. Chromatographic methods, like GC-MS, HPLC with UV-visible or fluorescence detector etc., are time-consuming, expensive and require a separation procedure before analysis. Because of the strong intrinsic fluorescence emission of PAHs, fluorescence spectroscopy could provide a highly sensitive method for the analysis of PAHs.

Synchronous fluorescence spectroscopy (SFS) has been known to be a simple and selective method for the simultaneous determination of multicomponent samples, ${ }^{2-6}$ while maintainning the fluorometric sensitivity. Inman and Winefordner ${ }^{7,8}$ described a kind of synchronous scanning mode, namely constant-energy synchronous luminescence spectrometry (CESLS), in which a constant energy difference $(\Delta \bar{v})$ between excitation and emission monochromators is maintained as each is scanned through the spectral region of interest. This approach has been successfully applied in PAH analysis. $^{9-18}$ In CESLS, the synchronous scanning can be optimized for a specific absorption-fluorescence transition by setting a $\Delta \bar{v}$ value equal to the vibrational energy difference. CESLS is more effective for narrowing the spectral band and reducing Raman scattering interference than general SFS. The

$\dagger$ To whom correspondence should be addressed.

E-mail: yqlig@xmu.edu.cn fluorescence excitation and emission spectra of PAHs are generally characterized with similar spaced vibrational features. CESLS can attain the same spectral features with a single scan as conventional constant-wavelength synchronous fluorescence spectroscopy in PAH multicomponent analysis.

However, resonance electronic energy transfer in mixture of PAHs has limited the application of SFS for PAH analysis. ${ }^{19}$ For example, there is appreciable energy transfer from pyrene to perylene in a solution containing both. ${ }^{20,21}$ The micellization of a PAHs mixture solution can help resolve this problem by isolating fluorophores in different micelles. ${ }^{22,23}$ Micelles can also easily solubilize PAHs and increase the fluorescence intensities of fluorophores, thereby increasing the sensitivity. The analysis of certain PAHs mixtures in micellar media has been made using conventional fluorometric techniques,,$^{22,24,25}$ as well as constant-wavelength and variable-angle synchronous fluorometry. $23,26,27$ It can be expected that CESLS would encounter the same problem of energy transfer, and micelles would be a good solution, as in other fluorometric methods.

In this paper, combined use of micellar media and constantenergy synchronous fluorescence spectroscopy for the simultaneous determination of pyrene, perylene and benzo $[a]$ pyrene in water samples is described. To our knowledge, this is the first report on the application of micellar media in CESLS. The method exempted the resonance electronic energy transfer among the different PAHs, and thus allowed their simultaneous identification and quantitative determination via a single spectrum. Because the method does not require pre-separating these compounds and dealing with much data, the analysis is simple and fast. 
Table 1 Maximal excitation and emission wavelengths $(\mathrm{nm})$ for the three PAHs in different media

\begin{tabular}{|c|c|c|c|c|c|c|c|c|c|c|c|c|c|c|c|c|c|c|}
\hline \multirow{3}{*}{ PAH } & \multicolumn{12}{|c|}{ Surfactant } & \multirow{2}{*}{\multicolumn{3}{|c|}{ Water }} & \multirow{2}{*}{\multicolumn{3}{|c|}{ Cyclohexane }} \\
\hline & \multicolumn{3}{|c|}{ CTAB } & \multicolumn{3}{|c|}{ SDBS } & \multicolumn{3}{|c|}{ SDS } & \multicolumn{3}{|c|}{$\mathrm{OP}$} & & & & & & \\
\hline & $\lambda_{\mathrm{ex}}$ & $\lambda_{\mathrm{em} 1}$ & $\lambda_{\mathrm{em} 2}$ & $\lambda_{\mathrm{ex}}$ & $\lambda_{\mathrm{em} 1}$ & $\lambda_{\mathrm{em} 2}$ & $\lambda_{\mathrm{ex}}$ & $\lambda_{\mathrm{em} 1}$ & $\lambda_{\mathrm{em} 2}$ & $\lambda_{\mathrm{ex}}$ & $\lambda_{\mathrm{em} 1}$ & $\lambda_{\mathrm{em} 2}$ & $\lambda_{\mathrm{ex}}$ & $\lambda_{\mathrm{em} 1}$ & $\lambda_{\mathrm{em} 2}$ & $\lambda_{\mathrm{ex}}$ & $\lambda_{\mathrm{em} 1}$ & $\lambda_{\mathrm{em} 2}$ \\
\hline $\mathrm{BaP}$ & 386 & 404.7 & 429 & 385 & 402 & 425 & 383 & 402 & 426 & 383.2 & 404.4 & 428.5 & 383 & 402 & 425 & 382 & 402 & 426 \\
\hline Pyr & 332.5 & 369 & 390 & 332 & 367 & 386 & 332.2 & 368 & 387 & 332 & 369 & 389 & 316 & 370 & 389.5 & 316 & 370 & 389 \\
\hline Per & 409 & 435 & 469 & 411 & 434 & 467 & 409 & 438 & 468 & 410 & 433 & 468 & 409 & 435 & 467 & 409 & 435 & 464 \\
\hline
\end{tabular}

\section{Experimental}

\section{Reagents}

Stock solutions of pyrene (Pyr, 99\%, Aldrich), benzo $[a]$ pyrene (BaP, 97\%, Aldrich) and perylene (Per, analytical grade, Shanghai Reagent) were prepared in methanol (analytical grade).

The surfactants hexadecyltrimethylammonium bromide (CTAB), sodium dodecylsulfate (SDS), sodium dodecylbenzene sulfonate (SDBS) and polyethylene glycol octylphenyl ether (OP) were of analytical grade and prepared in doubly deionized water.

\section{Apparatus}

All spectra were obtained on a laboratory-constructed, microcomputer-controlled spectrofluorometer, ${ }^{12-14}$ equipped with a $350 \mathrm{~W}$ xenon lamp and excitation and emission grating monochromators. The apparatus can be used to measure the fluorescence excitation spectra, emission spectra, all kinds of synchronous spectra (including constant-wavelength, constantenergy, and variable-angle) and their first or second-derivative spectra. An electronic differentiator was connected to the spectrofluorometer, and derivative spectra were recorded directly. Fluorescence spectra were obtained with the following instrumental parameters: both excitation and emission slits of 5 $\mathrm{nm}$ and a scan speed of $240 \mathrm{~nm} / \mathrm{min}$. All measurements were performed at room temperature.

\section{General procedure}

Fluorescence characteristics of PAHs in the surfactants. Working solutions were prepared by adding appropriate quantities of PAHs to different surfactant media, giving a methanol percentage smaller than $3 \%$. The surfactant concentration ranged over $1 \times 10^{-4}-0.08 \mathrm{M}$ for SDS, $1 \times 10^{-3}-$ $7 \times 10^{-3} \mathrm{M}$ for SDBS, $2 \times 10^{-3}-1.2 \times 10^{-2} \mathrm{M}$ for CTAB, $1 \times$ $10^{-5}-8 \times 10^{-4} \%$ for OP.

Determination of PAHs in water samples. The water samples (from seawater and lake water), after filtration, were spiked with four different PAHs concentrations, and their fluorescence intensities were measured in the presence of SDS micellar solutions.

\section{Results and Discussion}

\section{Selection of surfactant}

The excitation and emission spectral characteristics of pyrene, perylene and benzo $[a]$ pyrene were determined in an aqueous medium containing less than $3 \%$ methanol and in the presence of cationic, anionic and nonionic surfactants. The concentrations of the three PAHs were all $40 \mathrm{ng} \mathrm{ml}^{-1}$. As can be seen from Table 1, these PAHs show no obvious differences for
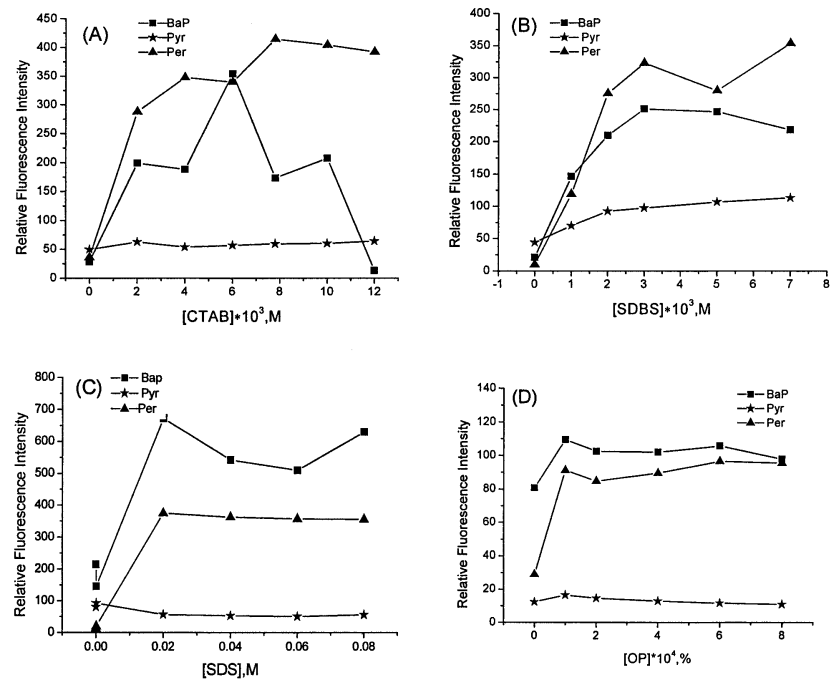

Fig. 1 Variation of the relative fluorescence of $(\boldsymbol{\square})$ benzo[ $a]$ pyrene (A) perylene and ( $\star$ ) pyrene with (A) CTAB, (B) SDBS, (C) SDS and (D) OP concentrations.

the maximal excitation and emission wavelengths in the different media, except a slight change in the $\lambda_{\mathrm{ex}}$ values of pyrene.

At the maximal excitation and emission wavelength of individual substances in different surfactants (shown in Table 1), the influence of the surfactant type and concentration upon the relative fluorescence of PAHs was investigated (Fig. 1). In the presence of CTAB, SDS, SDBS and OP, the three compounds showed different fluorescent behaviors. The fluorescence signals of perylene and benzo[a]pyrene increased rapidly, and then reached a constant value as the surfactant concentrations increased in most cases, while that of pyrene holds a general constant value as the surfactant concentration increased.

Comparing the behaviors of the PAHs in different surfactants, SDS could give a highest enhancement factor of the fluorescence intensity. The SDS micellar system at $0.02 \mathrm{M}$ was selected to perform the fluorometric determination of the three PAHs.

The fluorescence emission spectra of perylene, benzo $[a]$ pyrene and perylene in SDS micelle are shown in Fig. 2. These three PAHs could not be determined by conventional emission spectra, since their peaks overlapped.

\section{Selection of the constant-energy difference $(\Delta \bar{v})$}

The key experimental parameter in establishing a measurement procedure in CESLS is the so-called constantenergy difference (wavenumber interval), $\Delta \bar{v}$, which can be expressed as

$$
\Delta \bar{v}=\left(\frac{1}{\lambda_{\mathrm{ex}}}-\frac{1}{\lambda_{\mathrm{em}}}\right) \times 10^{7},
$$




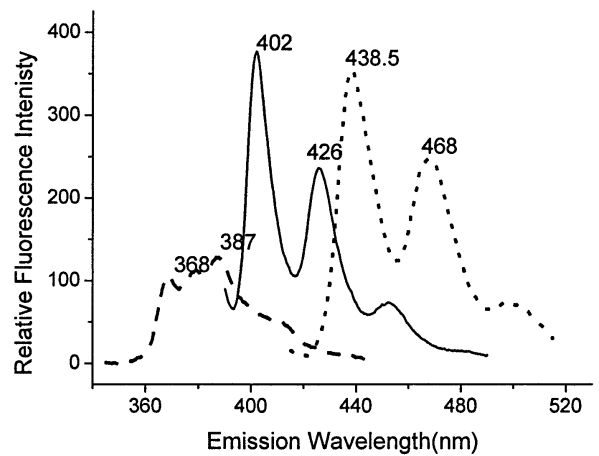

Fig. 2 Fluorescence emission spectra of $\operatorname{Pyr}\left(\cdots \cdots, \lambda_{\text {ex }}=332 \mathrm{~nm}\right)$, $\mathrm{BaP}\left(-, \lambda_{\mathrm{ex}}=383 \mathrm{~nm}\right)$ and Per $\left(\cdots \cdots \cdots \cdots, \lambda_{\mathrm{ex}}=409 \mathrm{~nm}\right)$ in $0.02 \mathrm{M}$ SDS.
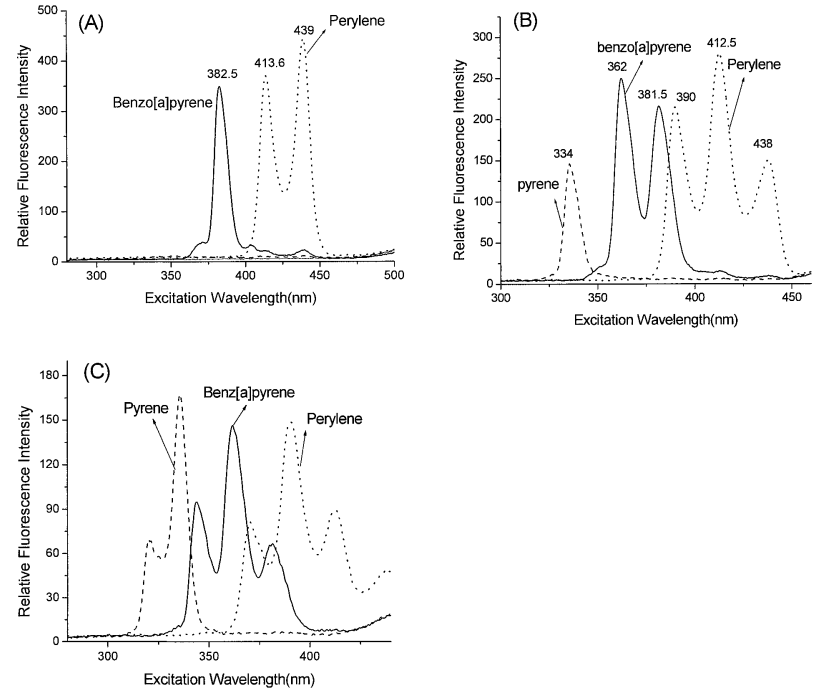

Fig. 3 Constant-energy synchronous spectra of pyrene, perylene and benzo $[a]$ pyrene in the presence of $0.02 \mathrm{M}$ SDS. (A) $\Delta \bar{v}=1300$ $\mathrm{cm}^{-1}$, (B) $\Delta \bar{v}=2800 \mathrm{~cm}^{-1}$, (C) $\Delta \bar{v}=4200 \mathrm{~cm}^{-1}$.

where $\lambda_{\mathrm{ex}}(\mathrm{nm})$ and $\lambda_{\mathrm{em}}(\mathrm{nm})$ are the excitation and emission wavelengths, respectively. The selection of this parameter was commonly empirical. Constant-energy differences of 1300, 2800 and $4200 \mathrm{~cm}^{-1}$, corresponding to one, two and three vibrational quanta, were compared (shown in Fig. 3).

For $\Delta \bar{v}=1300 \mathrm{~cm}^{-1}$ (Fig. 3(A)), the spectra of benzo[a]pyrene and perylene showed single and double peaks, respectively, whereas pyrene had no peak. For $\Delta \bar{v}=4200 \mathrm{~cm}^{-1}$ (Fig. 3(C)), the spectra of benzo[ $a]$ pyrene and perylene overlapped. When the constant-energy difference was $2800 \mathrm{~cm}^{-1}$, the spectra of pyrene, benzo $[a]$ pyrene and perylene revealed single, double and ternary peaks, respectively. Although benzo[ $a]$ pyrene and perylene overlapped at 381 and $390 \mathrm{~nm}$, simultaneous identification and determination of the three compounds could still be achieved by using their interference-free bands. Thus, a constant-energy difference of $2800 \mathrm{~cm}^{-1}$ was ultimately selected.

\section{Synchronous spectral characteristic in different media}

Figure 4 shows the CESL spectra of the three PAHs in micelles, non-micellar aqueous media and organic solvent when the constant-energy difference was set at $2800 \mathrm{~cm}^{-1}$. The fluorescence intensities of the PAHs in SDS were higher than
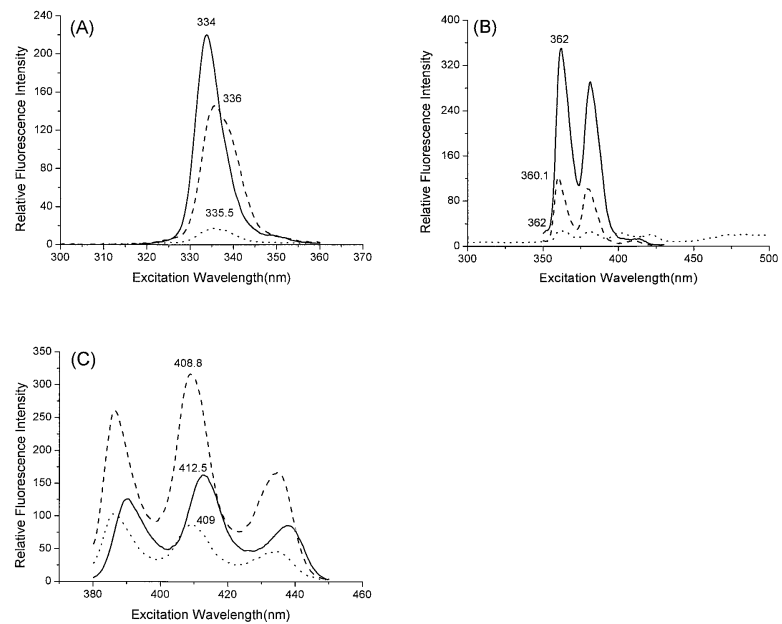

Fig. 4 Constant-energy synchronous spectra of (A) pyrene, (B) benzo $[a]$ pyrene and $(\mathrm{C})$ perylene in the presence of $0.02 \mathrm{M}$ SDS $(\cdots \cdots)$, cyclohexane $(-)$ and water $(\cdots \cdots \cdots \cdots) . \Delta \bar{v}=2800 \mathrm{~cm}^{-1}$.
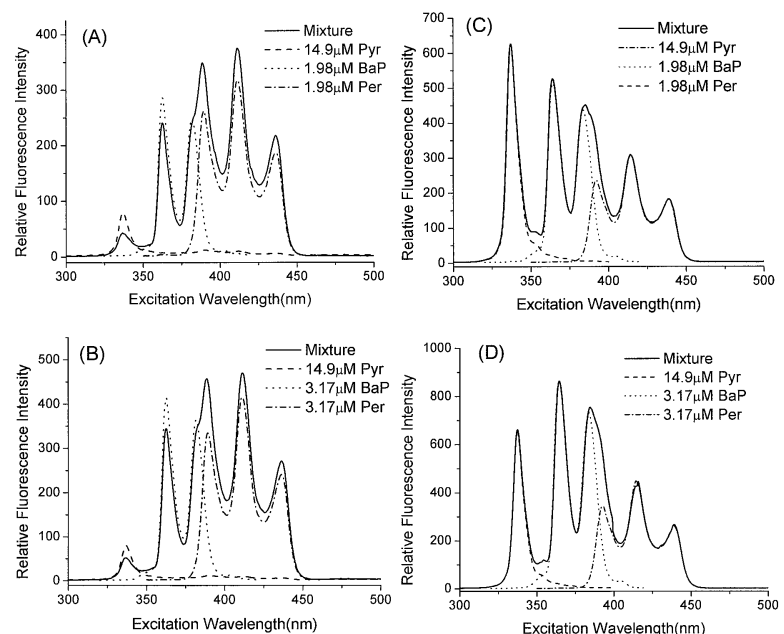

Fig. 5 Constant-energy synchronous spectra of pyrene, benzo $[a]$ pyrene, perylene and their mixture in cyclohexane ((A), (B)) and in $0.02 \mathrm{M}$ SDS ((C), (D)) with different concentration ratios of the three PAHs. The concentrations of the PAHs in the mixture were the same as those in a single component. $\Delta \bar{v}=2800 \mathrm{~cm}^{-1}$.

those in the rest media in most cases. Micelles confined and stabilized the PAH excited singlet states, thus increasing the fluorescence intensities..$^{20,26}$ The results indicate that a higher sensitivity should be obtained by using micellar media.

Figure 5 shows a comparison of the CESL spectra of pyrene, benzo $[a]$ pyrene and perylene in mixture and in a singlecomponent form when either cyclohexane or 0.02 M SDS were used as a solvent. It can be obviously observed that the fluorescence intensities of pyrene and benzo $[a]$ pyrene were reduced and that of perylene increased in cyclohexane, as shown in Figs. 5(A) and (B). This indicates an appreciable resonance electronic energy transfer of the PAHs in cyclohexane. The fluorescence emission bands of the donors (pyrene and benzo $[a]$ pyrene) showed an overlap with the absorption band of the acceptor (perylene). In this case, electronic energy transfer occurred from pyrene and benzo $[a]$ pyrene as donors (whose fluorescence was efficiently quenched) to perylene as an acceptor. However, on the 
Table 2 Analytical figures of merit with the constant-energy synchronous fluorescence technique

\begin{tabular}{|c|c|c|c|c|c|c|c|}
\hline \multirow{2}{*}{ Compound } & \multirow{2}{*}{$\begin{array}{l}\text { Linear range/ } \\
\text { ng ml-1 }\end{array}$} & \multicolumn{2}{|c|}{ Calibration graph ${ }^{\mathrm{a}}$} & \multicolumn{2}{|c|}{ Blank signal $^{\text {b }}$} & \multirow{2}{*}{$I_{\mathrm{F}} / I_{\mathrm{o}}^{\mathrm{c}}$} & \multirow{2}{*}{$\mathrm{LOD}^{\mathrm{d}} / \mathrm{ng} \mathrm{ml}^{-1}$} \\
\hline & & $I=a C+b$ & $r$ & Mean & S.D. & & \\
\hline Pyr & $2-1000$ & $I=3.15 C+3.46$ & 0.9997 & 4.7 & 0.74 & 12.8 & 0.70 \\
\hline $\mathrm{BaP}$ & $2-800$ & $I=3.38 C+2.43$ & 0.9997 & 4.9 & 0.72 & 12.3 & 0.63 \\
\hline Per & $2-1000$ & $I=6.6 C-63.19$ & 0.9999 & 4.7 & 0.75 & 2.0 & 0.34 \\
\hline
\end{tabular}

a. $I$, relative fluorescence intensity; $C$, concentration in $\mathrm{ng} \mathrm{ml}^{-1} ; a$ and $b$, constants. b. The mean value is based on eleven blank measurements. S.D. represents the standard deviation of blank signal. c. $I_{\mathrm{F}}$, relative fluorescence intensity in micellar medium; $I_{\mathrm{o}}$, relative fluorescence intensity in non-micellar aqueous medium. d. Limit of detection, concentration giving a signal three times the standard deviation of blank measurements.

Table 3 Determination of three compounds in artificial samples

\begin{tabular}{|c|c|c|c|c|c|c|c|c|c|}
\hline \multirow{2}{*}{ Sample } & \multicolumn{3}{|c|}{$\begin{array}{c}\text { Amount known/ } \\
\mathrm{ng} \mathrm{ml^{-1 }}\end{array}$} & \multicolumn{3}{|c|}{$\begin{array}{l}\text { Amount found/ } \\
\text { ng ml }{ }^{-1}\end{array}$} & \multicolumn{3}{|c|}{$\begin{array}{c}\text { Relative error, } \\
\%\end{array}$} \\
\hline & Pyr & $\mathrm{BaP}$ & Per & Pyr & $\mathrm{BaP}$ & Per & Pyr & $\mathrm{BaP}$ & Per \\
\hline 1 & 20.0 & 80.0 & 15.0 & 19.8 & 79.8 & 14.9 & -1.00 & -0.25 & -0.60 \\
\hline 2 & 35.0 & 65.0 & 30.0 & 38.0 & 64.7 & 29.6 & 0.0 & -0.46 & -1.3 \\
\hline 3 & 50.0 & 45.0 & 55.0 & 48.8 & 44.9 & 54.0 & -2.4 & -0.20 & -1.8 \\
\hline 4 & 65.0 & 30.0 & 65.0 & 64.6 & 29.3 & 62.0 & -0.6 & -2.3 & -4.60 \\
\hline 5 & 80.0 & 15.0 & 70.0 & 79.5 & 14.8 & 71.0 & -0.62 & -1.3 & 1.4 \\
\hline 6 & 95.0 & 20.0 & 80.0 & 94.8 & 19.6 & 79.0 & -0.20 & -2.0 & -1.2 \\
\hline
\end{tabular}

contrary, this case did not occur in the presence of SDS, as shown in Figs. 5(C) and (D). Though the concentrations of the three PAHs were high and the concentration ratios change in Figs. 5(C) and (D), the fluorescence signals of the three PAHs in either mixture or in pure form were identical, and there was no electronic energy transfer to be observed. The three PAHs in a mixture could therefore be quantitatively measured by using a single CESL spectrum in an SDS medium.

The hydrophobic PAHs molecules could be easily incorporated into micelles. A Poisson statistical law could be used to show the distribution of fluorescent molecules in a single micelle as ${ }^{20,23}$

$$
P(n)=x^{\mathrm{n}} e^{-\mathrm{x}} / n !
$$

Here $P(n)$ is the probability of " $n$ " fluorescent molecules appearing in the same micelle unit, and " $x$ " is the average number of fluorescent molecules per micelle. We took the mixture system of Fig. 5(D) as an example. The mixture contained $0.02 \mathrm{M}$ SDS and a total concentration of $21.14 \mu \mathrm{M}$ for pyrene, benzo $[a]$ pyrene and perylene. The typical aggregate number of SDS micelles is 62. ${ }^{28}$ Thus, the calculated probability for one PAH molecule in one micelle is 0.0655 and that of two PAH molecules in one micelle is 0.00201. This indicates that the chances of occupying the same micelle by two or more PAH molecules is negligible and, as a result, the PAHs molecules are segregated from each other even in the given high PAH concentrations. Thus, an electronic energy transfer of the PAHs is difficult to occur in SDS micelles

\section{Analytical figures of merit with CESLS}

Because of the high spectral resolution of CESLS, only a single scan is needed for the simultaneous identification and quantitative determination of these three components. The strongest spectral peak for each compound was used for quantification. The linear ranges, calibration graphs, blank
Table 4 Determination of the three PAHs in water samples

\begin{tabular}{|c|c|c|c|c|c|}
\hline \multirow[b]{2}{*}{$\mathrm{PAH}$} & \multirow{2}{*}{$\begin{array}{l}\text { Added/ } \\
\text { ng ml-1 }\end{array}$} & \multicolumn{2}{|c|}{ Seawater } & \multicolumn{2}{|c|}{ Lake water } \\
\hline & & $\begin{array}{l}\text { Found/ } \\
\mathrm{ng} \mathrm{ml}^{-1}\end{array}$ & $\begin{array}{c}\text { Recovery, } \\
\%\end{array}$ & $\begin{array}{l}\text { Found/ } \\
\mathrm{ng} \mathrm{ml}^{-1}\end{array}$ & $\begin{array}{c}\text { Recovery, } \\
\%\end{array}$ \\
\hline \multirow[t]{4}{*}{ Pyr } & 15.0 & 14.8 & 98.6 & 14.5 & 96.6 \\
\hline & 25.0 & 24.6 & 98.4 & 24.3 & 97.2 \\
\hline & 40.0 & 39.2 & 98.0 & 38.6 & 96.5 \\
\hline & 55.0 & 56.0 & 101.0 & 54.5 & 99.0 \\
\hline \multicolumn{3}{|c|}{ Average recovery $\pm \mathrm{SD}, \%$} & $99.0 \pm 1.36$ & & $97.3 \pm 1.16$ \\
\hline \multirow[t]{4}{*}{$\mathrm{BaP}$} & 55.0 & 54.6 & 99.0 & 54.2 & 99.0 \\
\hline & 40.0 & 39.8 & 99.5 & 39.1 & 97.7 \\
\hline & 30.0 & 28.9 & 96.3 & 28.2 & 94.0 \\
\hline & 25.0 & 24.7 & 98.8 & 24.5 & 98.0 \\
\hline \multicolumn{3}{|c|}{ Average recovery $\pm \mathrm{SD}, \%$} & $98.4 \pm 1.43$ & & $97.1 \pm 2.19$ \\
\hline \multirow[t]{4}{*}{ Per } & 20.0 & 19.6 & 98.0 & 19.5 & 97.5 \\
\hline & 35.0 & 35.2 & 100.0 & 34.5 & 98.5 \\
\hline & 50.0 & 51.2 & 102.0 & 52.0 & 104 \\
\hline & 65.0 & 64.8 & 99.6 & 64.6 & 99.3 \\
\hline \multicolumn{3}{|c|}{ Average recovery $\pm \mathrm{SD}, \%$} & $99.9 \pm 1.65$ & & $99.8 \pm 2.88$ \\
\hline
\end{tabular}

signals and detection limits were obtained as analytical characteristics of the fluorometric determination of PAHs in SDS as presented in Table 2. The enhancement factors of the fluorescence intensity in $0.02 \mathrm{M}$ SDS with respect to a nonmicellar aqueous medium $\left(I_{\mathrm{F}} / I_{\mathrm{O}}\right)$ are also shown in the table. The fluorescence signals were enhanced greatly for the three PAHs, especially for benzo $[a]$ pyrene and pyrene, in SDS micellar medium.

The precision of the method, expressed as relative standard deviation, was obtained by calculating 10 independent measurements. The used concentrations of the PAHs were 30 $\mathrm{ng} \mathrm{ml} l^{-1}$ for benzo[a]pyrene and perylene, and $50 \mathrm{ng} \mathrm{ml}^{-1}$ for pyrene. The relative standard deviations (RSD) were $1.7 \%$ for pyrene, $2.3 \%$ for benzo[a]pyrene and $2.5 \%$ for perylene, respectively.

\section{Determination of the three PAHs in artificial samples}

A mixture of benzo[a]pyrene, perylene and pyrene was used to examine the spectral overlapping interference. The concentration was set as follows: when the concentration of one compound was maximal, that of another whose spectral band was adjacent to this component was minimal. For a quantitative analysis, no mutual interference for any compound was found by using the proposed measurement method. Table 3 summarizes the determination results of three compounds in artificial samples. 
Determination of the three PAHs in water samples

The proposed method was applied to the determination of pyrene, perylene and benzo[a]pyrene in water samples from different origins, precisely spiked with four different amounts of the PAHs. Table 4 lists the added quantities, the amounts found and the obtained recovery percentages along with standard deviation. As shown in the table, a good agreement between the spiked and found quantities was observed. The recovery percentages were higher than $94 \%$ in all cases, with average recoveries of better than $97.1 \%$ for each compound.

\section{Conclusion}

The combination of the CESLS technique and a micellar system allows the simultaneous identification and quantitative determination of the PAHs by increasing the selectivity for individual PAHs present in a mixture, and eliminating the electronic energy transfer among PAHs. The proposed method is rapid and simple. Micelle-sensitized CESLS would extend the applicability of synchronous fluorescence spectroscopy.

\section{Acknowledgements}

We thank the Educational Ministry Foundation of China, the National Natural Science Foundation of China, the Natural Science Foundation of Fujian Government, and the Science and Technology Project of Xiamen for their financial support.

\section{References}

1. T. Vo-Dinh (ed.), "Chemical Analysis of Polycyclic Aromatic Compounds," 1989, Wiley-Interscience, New York.

2. T. Vo-Dinh and P. R. Martinez, Anal. Chim. Acta, 1981, 125, 13.

3. M. J. L. Villaizan, J. S. Lonzano, and M. A. L. Yusty, Talanta, 1995, 42, 967.

4. M. J. L. Villaizan, M. S. Garcia Falcon, S. Gonzalez Amogo, J. S. Lanzo, and M. A. L. Yusty, Talanta, 1996, 43, 1405 .

5. S. G. Falcon, S. G. Amigo, M. A. L. Yusty, M. J. L.
Villaizan, and J. S. Lozana, Talanta, 1996, 43, 659.

6. J. L. Vilchez, M. D. Olmo, R. Avidad, and L. F. CapitanVallvey, Analyst, 1994, 119, 1211.

7. E. L. Inman and J. D. Winefordner, Anal. Chim. Acta, 1982, 138, 245.

8. E. L. Inman and J. D. Winefordner, Anal. Chem., 1982, 54, 2018.

9. M. J. Kerkhoff, T. M. Lee, E. R. Allen, D. A. Lundgren, and J. D. Winefordner, Environ. Sci. Technol., 1985, 19 , 695.

10. M. J. Kerkhoff and J. D. Winefordner, Anal. Chim. Acta, 1985, 175, 257.

11. L. A. Files, B. T. Jones, S. Hanamura, and J. D. Winefordner, Anal. Chem., 1986, 58, 1440.

12. Y. Q. Li and X. Z. Huang, Chin. J. Anal. Chem., 1990, 18, 827.

13. Y. Q. Li, X. Z. Huang, J. G. Xu, and G. Z. Chen, Anal. Chim. Acta, 1992, 256, 285

14. Y. Q. Li, N. Shi, and F. Qian, Chem. J. Chin. Univ., 1997, 18, 538.

15. A. A. Eiroa, S. R. Huckins, and E. V. Blanco, Appl. Spectrosc., 2000, 54, 1534

16. A. A. Eiroa, E. V. Blanco, and P. L. Machia, Analyst, 2000 , $125,1321$.

17. A. A. Eiroa, E. V. Blanco, P. L. Mahia, S. M. Lorenzo, D. P. Rodriguez, and E. F. Fernandez, Talanta, 2000, 51, 677.

18. J. A. M. Pulgarin and A. A. Eiroa, Talanta, 1994, 41, 21.

19. A. A. Eiroa, E. V. Blanco, P. L. Mahia, S. M. Lorenzo, and D. P. Rodriguez, Analyst, 1998, 123, 2113.

20. D. Patra and A. K. Mishra, Anal. Lett., 2000, 33, 2293.

21. A. Sharma and O. S. Wolfbeis, Appl. Spectrosc., 1988, 42, 1009.

22. E. Lazaro, M. P. San Andres, and S. Vera, Anal. Chim. Acta, 2000, 413, 159.

23. D. Patra and A. K. Mishra, Talanta, 2001, 55, 143.

24. J. Amador-Hemandez, A. Cladera, E. M. Estela, P. L. Lopez-de-Alba, and V. Cerda, Analyst, 1998, 123, 2235.

25. J. L. Beltran, R. Ferrer, and J. Guiteras, Anal. Chim. Acta, 1998, 373, 311 .

26. D. Patra, Luminescence, 2003, 18, 97.

27. Y. Q. Li and L. Shi, Chin. J. Anal. Chem., 1996, 24, 41.

28. L. J. Cline Love and J. G. Dorsey, Anal. Chem., 1984, 56, 1132A. 